BU-HEPP-05/03

CASPER-05/03

hep-ph/0506183

June 2005

\title{
Observable/Hidden Sector Broken Symmetry for Symmetric Boundary Conditions
}

\author{
B. Dundee, ${ }^{*}$ J. Perkins,${ }^{\dagger}$ and G. Cleaver,$^{\dagger}$ \\ Center for Astrophysics, Space Physics 83 Engineering Research, \\ Department of Physics, Baylor Science Building, Baylor University, \\ Waco, TX 76706, USA
}

\begin{abstract}
A 4-dimensional heterotic string model of free fermionic construction is presented wherein mirror symmetry breaking between observable and hidden sector gauge groups occurs in spite of mirror symmetry between observable and hidden sector worldsheet fermion boundary conditions. The differentiation is invoked by an asymmetry in GSO projections necessarily resulting from the symmetry of the free fermionic boundary conditions. In the specific examples shown, an expected non-chiral Pati-Salam mirror universe model is transformed into a chiral model with enhanced hidden sector gauge symmetry and reduced observable sector gauge symmetry: $\left[S U(4)_{C} \otimes S U(2)_{L} \otimes\right.$ $\left.S U(2)_{R}\right]^{O} \otimes\left[S U(4)_{C} \otimes S U(2)_{L} \otimes S U(2)_{R}\right]^{H}$, is necessarily transformed into a chiral $\left[S U(4)_{C} \otimes S U(2)_{L}\right]^{O} \otimes\left[S O(10) \otimes S U(2)_{R}\right]^{H}$ model because of an unavoidable asymmetry in GSO projections.
\end{abstract}

\footnotetext{
*Ben_Dundee@baylor.edu

†John_Perkins@baylor.edu

${ }^{\ddagger}$ Gerald_Cleaver@baylor.edu
} 


\section{Mirror Universe Models}

Mirror universe models, with hidden sector matter and forces identical to that of the visible world, but interacting with the latter only via gravity, have been proposed in the context of neutrino physics (specifically by attempts to understand the nature of a sterile neutrino) and in the context of superstring/M-theory [1, 2, 3, 4, 5]. As a means to reconcile the constraints of big bang nucleosynthesis, the reheating temperature of the mirror universe after inflation was postulated to be lower than that in the observable universe [1]. From this it was shown that the asymmetric reheating can be related to a difference of the electroweak symmetry breaking scales in the two sectors, as required for a mirror solution to the neutrino puzzle. In such models it was shown that the baryon asymmetry is greater in the mirror universe than in the observable universe and that the mirror baryons could provide the dominant dark matter in the bulk universe [4].

While heterotic strings (M-theory) offer a mirror universe scenario in ten (eleven) dimensions, with the well-known $E_{8}$ gauge symmetry for both observable and hidden sectors (9-branes), string-derived quasi-realistic three generation mirror universe models in four-dimensions have, to our knowledge, not been constructed. Rather, in typical quasi-realistic string models, the ten-dimensional mirror symmetry is broken through compactification to four dimensions. In bosonic lattice/orbifold construction differentiation of observable and hidden gauge groups (and, thus, of matter representations) is typically a result of asymmetric Wilson loop effects and spin embedding. In corresponding free fermionic [9] models, mirror symmetry breaking generally results from asymmetric boundary conditions between observable and hidden sector worldsheet fermions. An investigation into string/M-derived mirror models is underway and will be reported in an upcoming paper [6]. Contrastingly, in this letter we wish to discuss an obstacle to mirror model construction that can develop in weakly coupled heterotic strings. This obstacle, nonetheless, produces an interesting physical effect in its own right. In this letter we will demonstrate how, under a specific set of conditions, mirror symmetry breaking will necessarily occur in models with symmetric boundary conditions. We will investigate the implications of this for initially mirror symmetric models.

During our string/M-derived mirror universe investigation, we found that even when symmetric worldsheet fermion boundary conditions are imposed, breaking of mirror symmetry is sometimes mandated by an unavoidable asymmetry imposed by the GSO projections. In this note we show, through two example models, how GSO projections can necessitate mirror symmetry breaking of observable $(O)$ and hidden sector $(H)$ Pati-Salam [7, 8] gauge groups,

$$
\left[S U(4)_{C} \otimes S U(2)_{L} \otimes S U(2)_{R}\right]^{O} \otimes\left[S U(4)_{C} \otimes S U(2)_{L} \otimes S U(2)_{R}\right]^{H} .
$$

In our first example, the GSO projections reduce the observable sector gauge group 


$$
\left[S U(4)_{C} \otimes S U(2)_{L}\right]^{O}
$$

by transferring $S U(2)_{R}^{O}$ to the hidden sector. In the process the

$$
\left[S U(4)_{C} \otimes S U(2)_{L}\right]^{H}
$$

subgroup of the hidden sector Pati-Salam gauge symmetry is enhanced to

$$
[S O(10)]^{H}
$$

Additionally, the initial shadow $(S)$ sector (corresponding to charges possibly carried by both observable and hidden states) gauge group, $\left[S U(2)^{3} \otimes S U(3) \otimes U(1)^{7}\right]^{S}$, absorbs $\left[S U(2)_{R}\right]^{H}$ to become $\left[S U(2)^{3} \otimes S U(5) \otimes U(1)^{6}\right]^{S}$ (see Table B.2).

Our second example differs from the first in some of the elements of its GSO projections matrix. For model 2, the GSO projections reduce the observable sector Pati-Salam gauge group to $\left[S U(4)_{C} \otimes S U(2)_{R}\right]^{O}$ by alternately transferring $S U(2)_{L}^{O}$ to the hidden sector. The hidden sector $\left[S U(4)_{C} \otimes S U(2)_{R}\right]^{H}$ subgroup of the PatiSalam group is similarly enhanced to $[S O(10)]^{H}$. In this version, the shadow sector gauge group remains of rank 12 and does not absorb $\left[S U(2)_{L}\right]^{H}$ (see Table C.2).

In Section 2 we briefly review free fermionic construction and the related form of the GSO projections. In Section 3 we then review the NAHE-based $\mathbb{Z}_{2} \times \mathbb{Z}_{2}$ models [10], the class to which our example models belong (see Table A.1). Following this, in Section 4 we introduce the mirror-symmetric free fermion worldsheet boundary conditions for our two models (see Tables A.2 and A.3). We then demonstrate how an unavoidable asymmetry that arises between certain GSO projection matrix elements in our models produces mirror symmetry breaking between observable and hidden sector states. We study viable hypercharge definitions in Section 5. Our investigation of the unavoidable observable/hidden sector mirror-breaking effect in our models is then summarized in Section 6. We present the gauge group and matter states for our first model in Tables B.2 and B.3, respectively, and those for our second model in Tables C.2 and C.3, respectively.

\section{Free Fermionic Models}

A free fermionic heterotic string model is specified by two objects [9]. The first is a $p$-dimensional basis set of free fermionic boundary vectors $\left\{\mathbf{V}_{i}, i=1, \ldots, p\right\}$. Each vector $\mathbf{V}_{i}$ has 64 components, $-1<V_{i}^{m} \leq 1, m=1, \ldots, 64$, with the first 20 components specifying boundary conditions for the 20 real free fermions representing worldsheet degrees of freedom for the left-moving supersymmetric string, and the later 44 components specifying boundary conditions for the 44 real free fermions representing worldsheet degrees of freedom for the right-moving bosonic string. (Components of 
$\mathbf{V}_{i}$ for complex fermions are double-counted.) Modular invariance dictates that the basis vectors, $\mathbf{V}_{i}$, span a finite additive group $\Xi=\left\{\sum_{n_{i}=1}^{N_{i}-1} \sum_{i=1}^{p} n_{i} \mathbf{V}_{i}\right\}$, with $N_{i}$ the lowest positive integer such that $N_{i} \mathbf{V}_{i}=\mathbf{0}(\bmod 2) . \mathbf{V}_{1}=\mathbf{1}$ (a 64 -component unit vector) must be present in all models. In a given sector $\boldsymbol{\alpha} \equiv a_{i} \mathbf{V}_{i}(\bmod 2) \in \Xi$, with $a_{i} \in\left\{0,1, \ldots, N_{i}-1\right\}$, a worldsheet fermion $f_{m}$ transforms as $f_{m} \rightarrow-\exp \left\{\pi \alpha_{m}\right\} f_{m}$ around non-contractible loops on the worldsheet. Boundary vector components for real fermions are thus limited to be either 0 or 1 , whereas boundary vector components for complex fermions can be rational [9].

The second object necessary to define a free fermionic model (up to vacuum expectation values (VEVs) of fields in the effective field theory) is a $p \times p$-dimensional matrix $\mathbf{k}$ of rational numbers $-1<k_{i, j} \leq 1, i, j=1, \ldots, p$, that determine the GSO operators for physical states. The $k_{i, j}$ are related to the phase weights $C\left(\begin{array}{l}\mathbf{v}_{i} \\ \mathbf{v}_{j}\end{array}\right)$ appearing in a model's one-loop partition function $Z$ :

$$
C\left(\begin{array}{c}
\mathbf{V}_{i} \\
\mathbf{V}_{j}
\end{array}\right)=(-1)^{s_{i}+s_{j}} \exp \left(\pi i k_{j, i}-\frac{1}{2} \mathbf{V}_{i} \cdot \mathbf{V}_{j}\right),
$$

where $s_{i}$ is the 4-dimensional spacetime component of $\mathbf{V}_{i}$. (The inner product of boundary (or charge) vectors is lorentizian, taken as left-movers minus right movers. Contributions to inner products from real fermion boundary components are weighted by a factor of $\frac{1}{2}$ compared to contributions from complex fermion boundary components.)

The phase weights $C\left(\begin{array}{l}\boldsymbol{\alpha} \\ \boldsymbol{\beta}\end{array}\right)$ for general sectors

$$
\boldsymbol{\alpha}=\sum_{j=1}^{p} a_{j} \mathbf{V}_{j} \in \Xi, \quad \boldsymbol{\beta}=\sum_{i=1}^{p} b_{i} \mathbf{V}_{i} \in \Xi
$$

can be expressed in terms of the components in the $p \times p$-dimensional matrix $\mathbf{k}$ for the basis vectors:

$$
C\left(\begin{array}{c}
\boldsymbol{\alpha} \\
\boldsymbol{\beta}
\end{array}\right)=(-1)^{s_{\alpha}+s_{\beta}} \exp \left\{\pi i \sum_{i, j} b_{i}\left(k_{i, j}-\frac{1}{2} \mathbf{V}_{i} \cdot \mathbf{V}_{j}\right) a_{j}\right\}
$$

Modular invariance simultaneously imposes constraints on the basis vectors $\mathbf{V}_{i}$ and on components of the GSO projection matrix $\mathbf{k}$ :

$$
\begin{aligned}
k_{i, j}+k_{j, i} & =\frac{1}{2} \mathbf{V}_{i} \cdot \mathbf{V}_{j}(\bmod 2) \\
N_{j} k_{i, j} & =0(\bmod 2) \\
k_{i, i}+k_{i, 1} & =-s_{i}+\frac{1}{4} \mathbf{V}_{i} \cdot \mathbf{V}_{i}(\bmod 2)
\end{aligned}
$$

The dependence upon the $k_{i, j}$ can be removed from equations (2.4-2.6), after appropriate integer multiplication, to yield three constraints on the $\mathbf{V}_{i}$ :

$$
N_{i, j} \mathbf{V}_{i} \cdot \mathbf{V}_{j}=0(\bmod 4)
$$




$$
N_{i} \mathbf{V}_{i} \cdot \mathbf{V}_{i}=0(\bmod 8)
$$

The number of real fermions simultaneously periodic

for any three basis vectors is even.

$N_{i, j}$ is the lowest common multiple of $N_{i}$ and $N_{j}$. (2.9) applies when two or more of three the basis vectors are identical. Thus, each basis vector must have an even number of real periodic fermions.

The physical massless states in the Hilbert space of a given sector $\boldsymbol{\alpha} \in \Xi$, are obtained by acting on the vacuum with bosonic and fermionic operators and by applying generalized GSO projections. The $U(1)$ charges for the Cartan generators of the unbroken gauge group are in one to one correspondence with the $U(1)$ currents $f_{m}^{*} f_{m}$ for each complex fermion $f_{m}$, and are given by:

$$
Q_{m}^{\boldsymbol{\alpha}}=\frac{1}{2} \alpha_{m}+F_{m}^{\boldsymbol{\alpha}},
$$

where $\alpha_{m}$ is the boundary condition of the worldsheet fermion $f_{m}$ in the sector $\boldsymbol{\alpha}$, and $F_{m}^{\boldsymbol{\alpha}}$ is a fermion number operator counting each mode of $f_{m}$ once and of $f_{m}^{*}$ minus once. Pseudo-charges for non-chiral (i.e., with both left- and right-moving components) real Ising fermions $f_{m}$ can be similarly defined, with $F_{m}$ counting each real mode $f$ once.

For periodic fermions, $\alpha_{m}=1$, the vacuum is a spinor representation of the Clifford algebra of the corresponding zero modes. For each periodic complex fermion $f_{m}$ there are two degenerate vacua $|+\rangle,|-\rangle$, annihilated by the zero modes $\left(f_{m}\right)_{0}$ and $\left(f_{m}^{*}\right)_{0}$ and with fermion numbers $F_{m}^{\boldsymbol{\alpha}}=0,-1$, respectively.

The boundary basis vectors $\mathbf{V}_{j}$ generate the set of GSO projection operators for physical states from all sectors $\boldsymbol{\alpha} \in \Xi$. In a given sector $\boldsymbol{\alpha}$, the surviving states are those that satisfy the GSO equations imposed by all $\mathbf{V}_{j}$ and determined by the $k_{j, i}$ 's:

$$
\mathbf{V}_{j} \cdot \mathbf{F}^{\boldsymbol{\alpha}}=\left(\sum_{i} k_{j, i} a_{i}\right)+s_{j}-\frac{1}{2} \mathbf{V}_{j} \cdot \boldsymbol{\alpha}(\bmod 2)
$$

or, equivalently,

$$
\mathbf{V}_{j} \cdot \mathbf{Q}^{\boldsymbol{\alpha}}=\left(\sum_{i} k_{j, i} a_{i}\right)+s_{j}(\bmod 2) .
$$

For a given set of basis vectors, the independent GSO matrix components are $k_{1,1}$ and $k_{i, j}$, for $i>j$. This GSO projection constraint, when combined with equations (2.42.6) form the free fermionic re-expression of the even, self-dual modular invariance constraints for bosonic lattice models. 


\section{$3 \quad \mathbb{Z}_{2} \times \mathbb{Z}_{2}$ NAHE-Based Models}

The $\mathbb{Z}_{2} \times \mathbb{Z}_{2}$ NAHE-based models have contributed much to understanding regions of the parameter space of weakly-coupled (quasi)-realistic string models, including GUT's [11], semi-GUT's [8, 12], near-MSSM's [13, 14], and MSSM's [15]. They have advanced knowledge of general features of classes of string model, both perturbative [16] and non-perturbative properties [17] that likely still persist after M-theory embedding. The NAHE set [10] consists of the five basis vectors, $\mathbf{1}, \mathbf{S}$, and $\mathbf{b}_{i}, i=1$ to 3, given in Table A.1. The NAHE set breaks the observable sector $E_{8}$ gauge symmetry to $S O(10) \otimes U(1)^{3}$ but does not affect the hidden sector $E_{8}$. The six compactified directions, represented by six pairs of left- and right-moving real fermions $\{y, \omega \mid \bar{y}, \bar{\omega}\}^{1, \cdots, 6}$, generate an additional $S O(12)$ shadow sector (S) gauge symmetry. The NAHE set breaks this to $S O(4)^{3}$. However, each $U(1)_{i}$ combines with a corresponding $S O(4)_{i}$ to form an enhanced $S O(6)_{i}$ symmetry. Thus, the gauge group of the NAHE set is $\left[S O(10) \otimes S O(6)^{3}\right]^{O} \otimes E_{8}^{H}$ with $N=1$ spacetime supersymmetry. The observable sector matter is 48 spinorial 16's of $S O(10)$, with sixteen of these from each sector $\mathbf{b}_{1}, \mathbf{b}_{2}$ and $\mathbf{b}_{3}$.

The $U(1)_{i}$, for $i=1$ to 3 , act as generation charges for the three sectors $\mathbf{b}_{1}, \mathbf{b}_{2}$ and $\mathbf{b}_{3}$, respectively. The sixteen copies of $\mathbf{1 6}$ 's of $S O(10)$ from a given $\mathbf{b}_{i}$ are formed from 4 copies of 4 's of $S O(6)_{i}$. To obtain three generation models, the $S O(6)^{3}$ must be broken to no more than the initial $U(1)^{3}$. Reduction of the $S O(10)$ and of the $S O(6)^{3}$ can result from additional boundary basis vectors that will break $S O(10)$ to one of its subgroups, $S U(5) \times U(1), S O(6) \times S O(4)$ or $S U(3) \times S U(2) \times U(1)^{2}$. The additional sectors must simultaneously reduce the number of generations to three, one from each of the sectors $\mathbf{b}_{1}, \mathbf{b}_{2}$ and $\mathbf{b}_{3}$. The detailed phenomenological properties of the various NAHE-based three generation models can differ significantly. In particular, the properties strongly depend on the boundary components of the non-NAHE basis vectors for the fermions representing the six compactified directions [18].

\section{Symmetry Breaking of Mirror Models}

Mirror models with matching observable and hidden sector symmetries and states may be created from NAHE-based models by adding mirror basis vectors, $\mathbf{b}_{1}^{\prime}, \mathbf{b}_{2}^{\prime}$ and $\mathbf{b}_{3}^{\prime}$, as defined in Table A.2. Due to the symmetry between $\mathbf{b}_{i}$ and $\mathbf{b}_{i}^{\prime}$, these mirror vectors break the hidden sector $E_{8}$ in the same manner as the NAHE set breaks the observable sector $E_{8}$ into $S O(10) \otimes U(1)^{3}$. Each $\mathbf{b}_{i}^{\prime}$ produces 16 copies of $\mathbf{1 6}$ 's of the hidden sector $S O(10)$.

The right-moving components of the $\mathbf{b}_{i}$ and the $\mathbf{b}_{i}^{\prime}$ basis vectors that are simultaneously non-zero form a subset of $\{y, \omega \mid \bar{y}, \bar{\omega}\}^{1, \cdots, 6}$. That is, $\mathbf{b}_{i}$ and $\mathbf{b}_{i}^{\prime}$ states will both carry some $\{y, \omega \mid \bar{y}, \bar{\omega}\}^{1, \cdots, 6}$ "shadow sector" charges. Within the NAHE set the only two bosonic sectors that can produce gauge states are $\mathbf{0}$ and $\mathbf{1}+\mathbf{b}_{1}+\mathbf{b}_{2}+\mathbf{b}_{3}$. All observable and "shadow sector" $S O(n)$ and $U(1)$ generators, along with the hidden 
sector 120 rep of $S O(16) \in E_{8}$, originate in $\mathbf{0}$, while the hidden sector $\mathbf{1 2 8}$ rep of $S O(16) \in E_{8}$ originates in $\mathbf{1}+\mathbf{b}_{1}+\mathbf{b}_{2}+\mathbf{b}_{3}$. The GSO projections from the mirror sectors $\mathbf{b}_{1}^{\prime}, \mathbf{b}_{2}^{\prime}$, and $\mathbf{b}_{3}^{\prime}$ remove the $\mathbf{1 2 8}$ rep of $S O(16)$, reducing the hidden sector $E_{8}$ symmetry to $S O(16)$. Further, the mirror sectors reduce the hidden sector $S O(16)$ to an $S O(10) \otimes U(1)^{3}$ symmetry, matching the observable sector. (Similarly the GSO projections of $\mathbf{b}_{1}, \mathbf{b}_{2}$, and $\mathbf{b}_{3}$ remove any contribution to the observable gauge group from the $\mathbf{1}+\mathbf{b}_{1}^{\prime}+\mathbf{b}_{2}^{\prime}+\mathbf{b}_{3}^{\prime}$ sector.)

The hidden sector $U(1)^{3}$ generation charges and the observable sector $U(1)^{3}$ generation charges combine with the shadow sector charges in a like manner. Simultaneously, the $\mathbf{b}_{i}^{\prime}$ sector GSO's reduce the $S O(4)^{3}$ shadow sector symmetry to $U(1)^{6}$. The net result is a $S U(3) \otimes S U(2)^{3} \otimes U(1)^{7}$ shadow sector symmetry, whose charges are carried by matter representations of the observable $S O(10)$ and of the hidden $S O(10)$. The additional $S U(3) \otimes S U(2)^{3}$ generators originate in several additional massless gauge sectors formed from linear combinations of $\mathbf{1}, \mathbf{b}_{i}$, and $\mathbf{b}_{i}^{\prime}$. All of these sectors have massless vacua that only carry $\bar{\eta}_{i}, \bar{\eta}_{i}^{\prime}$, and $\bar{y}, \bar{\omega}$ shadow charges. While most massless gauge states from these sectors are projected out, a few are not. Those that survive combine observable sector $\bar{\eta}$-charges, hidden sector $\bar{\eta}^{\prime}$-charges, and shadow sector $\bar{y}, \bar{\omega}$-charges (see Tables B.2 and C.2). Thus, at this stage mirror symmetry still exists, as should be expected. Note in particular that $\mathbf{k}$ is invariant under exchange of $\mathbf{b}_{i}$ with co responding $\mathbf{b}_{i}^{\prime}$.

To break each of the $S O(10)$ to their corresponding Pati-Salem $S U(4)_{C} \otimes S U(2)_{L} \otimes$ $S U(2)_{R}$, two additional sectors $\mathbf{a}$ and $\mathbf{a}^{\prime}$ are added to the model, where a and $\mathbf{a}^{\prime}$ are mirror sectors, as shown in in Table A.2. The set of 10 basis vectors $\left\{\mathbf{1}, \mathbf{S}, \mathbf{b}_{1}, \mathbf{b}_{2}, \mathbf{b}_{3}, \mathbf{b}_{1}^{\prime}, \mathbf{b}_{2}^{\prime}, \mathbf{b}_{3}^{\prime}, \mathbf{a}, \mathbf{a}^{\prime}\right\}$, produce a model with mirror symmetry boundary conditions for the observable and hidden sectors. However, with the addition of $\mathbf{a}$ and $\mathbf{a}^{\prime}$, an unavoidable asymmetry develops among the GSO projection components: Although all new degrees of freedom of $k_{\boldsymbol{\alpha}, \boldsymbol{\beta}}$, for $\boldsymbol{\alpha} \in\left\{\mathbf{a}, \mathbf{a}^{\prime}\right\}$ and $\boldsymbol{\beta} \in\left\{\mathbf{1}, \mathbf{S}, \mathbf{b}_{1}, \mathbf{b}_{2}, \mathbf{b}_{3}, \mathbf{b}_{1}^{\prime}, \mathbf{b}_{2}^{\prime}, \mathbf{b}_{3}^{\prime}\right\}$, are chosen to be invariant under simultaneous exchange of primed and un-primed sectors for both $\boldsymbol{\alpha}$ and $\boldsymbol{\beta}$, symmetry breaking between $k_{\mathbf{a}, \mathbf{a}^{\prime}}$ and $k_{\mathbf{a}^{\prime}, \mathbf{a}}$ occurs automatically. Only one of $k_{\mathbf{a}, \mathbf{a}^{\prime}}$ and $k_{\mathbf{a}^{\prime}, \mathbf{a}}$ is a degree of freedom; the other is specified by (2.4). As Table A.3 shows, $\mathbf{a} \cdot \mathbf{a}^{\prime}=10$, which from (2.4) yields

$$
k_{\mathbf{a}, \mathbf{a}^{\prime}}+k_{\mathbf{a}^{\prime}, \mathbf{a}}=1(\bmod 2) .
$$

Thus, $k_{\mathbf{a}, \mathbf{a}^{\prime}}$ and $k_{\mathbf{a}^{\prime}, \mathbf{a}}$ cannot be equal $(\bmod 2)$; either $k_{\mathbf{a}, \mathbf{a}^{\prime}}=1$ and $k_{\mathbf{a}^{\prime}, \mathbf{a}}=0$ or vice versa (since all components of $\mathbf{a}$ and $\mathbf{a}^{\prime}$ are either anti-periodic or periodic).

The addition of $\mathbf{a}$ and $\mathbf{a}^{\prime}$ to the model generates several new massless gauge sectors of the form $\mathbf{a}+\mathbf{a}^{\prime}+\ldots$. However, in both model variations presented herein, the GSO projections remove all possible gauge states from such sectors, except those coming from $\mathbf{a}+\mathbf{a}^{\prime}$. The $\mathbf{a}+\mathbf{a}^{\prime}$ sector has all anti-periodic components except for four periodic associated with the two complex fermions generating the observable $S O(4)=S U(2)_{L} \otimes S U(2)_{R}$ and the two complex fermions generating the hidden 
sector $S O(4)=S U(2)_{L} \otimes S U(2)_{R}$. Thus, in the $\mathbf{a}+\mathbf{a}^{\prime}$ sector, massless gauge states require one anti-periodic fermionic (with $Q= \pm 1$ ) excitation.

The GSO projection from a acts on observable $S O(4)$ spinors while $\mathbf{a}^{\prime}$ acts on hidden $S O(4)$ spinors. Since $k_{\mathbf{a}, \mathbf{a}^{\prime}}$ and $k_{\mathbf{a}, \mathbf{a}^{\prime}}$ differ by $1(\bmod 2)$, so do $k_{\mathbf{a}, \mathbf{a}+\mathbf{a}^{\prime}}$ and $k_{\mathbf{a}^{\prime}, \mathbf{a}+\mathbf{a}^{\prime}}$. Thus, a state in the $\mathbf{a}+\mathbf{a}^{\prime}$ sector survives both the a sector GSO projections and $\mathbf{a}^{\prime}$ sector GSO projections if and only if its observable and hidden $S O(4)$ spinors have opposite chirality. That is, the net number of down spins among the four spinors must be odd, implying that an $\mathbf{a}+\mathbf{a}^{\prime}$ gauge state will either carry observable $S U(2)_{L} \in S O(4)$ charge and hidden $S U(2)_{R} \in S O(4)$ charge or vice-versa.

For model 1, the additional $k_{\mathbf{b}_{i}^{\left({ }^{\prime}\right), \mathbf{a}}}$ and $k_{\mathbf{b}_{i}^{\left({ }^{\prime}\right)}, \mathbf{a}^{\prime}}$ require $\mathbf{a}+\mathbf{a}^{\prime}$ states to always have an even number of observable $S O(4)-\frac{1}{2}$ spins and an odd number of hidden $S O(4)-\frac{1}{2}$ spins, linking observable $S U(2)_{R}$ with hidden $S U(2)_{L}$. The remaining GSO projections on $\mathbf{a}+\mathbf{a}^{\prime}$ gauge states require the $Q= \pm 1$ anti-periodic fermion excitation charge to be from the hidden sector $S O(6) \sim S U(4)$. Thus, the surviving $\mathbf{a}+\mathbf{a}^{\prime}$ simple root connects observable $S U(2)_{R}$ roots with the hidden sector $S O(6)$ and $S U(2)_{L}$ roots, thereby regenerating a hidden sector $S O(10)$ (see Table B.2):

$$
\left[S U(2)_{R}\right]^{O} \otimes\left[S O(6) \otimes S U(2)_{L}\right]^{H} \rightarrow[S O(10)]^{H} .
$$

In addition, a sector $1+\mathbf{b}_{1}+\mathbf{b}_{1}^{\prime}+\mathbf{a}$ gauge state with eight complex spinors links the shadow gauge states with the hidden sector $S U(2)_{R}$, increasing the shadow sector gauge symmetry,

$$
\left[S U(3) \otimes S U(2)^{3} \otimes U(1)^{7}\right]^{S} \otimes\left(S U(2)_{R}\right)^{H} \rightarrow\left[S U(5) \otimes S U(2)^{3} \otimes U(1)^{6}\right]^{S} .
$$

The final model 1 gauge group is, therefore,

$$
\left[S U(4) \otimes S U(2)_{L}\right]^{O} \otimes\left[S U(5) \otimes S U(2)^{3} \otimes U(1)^{6}\right]^{S} \otimes[S O(10)]^{H} .
$$

While maintaining symmetry under exchange of primed and un-primed components of $\mathbf{k}$, model 2 differs from model 1 in some choices of $k_{\mathbf{a}, \beta}$ and $k_{\mathbf{a}^{\prime}, \beta}$, for $\boldsymbol{\beta} \in\left\{\mathbf{1}, \mathbf{S}, \mathbf{b}_{1}, \mathbf{b}_{2}, \mathbf{b}_{3}, \mathbf{b}_{1}^{\prime}, \mathbf{b}_{2}^{\prime}, \mathbf{b}_{3}^{\prime}\right\}$ (see Table C.1). Model 2 GSO projections require an odd number of observable $S O(4)-\frac{1}{2}$ spins and an even number of hidden $S O(4)-\frac{1}{2}$ spins in the $\mathbf{a}+\mathbf{a}^{\prime}$ sector. This link observable $S U(2)_{L}$ reps with hidden $S U(2)_{R}$ reps. The remaining GSO projections again require the $Q= \pm 1$ anti-periodic fermion excitation charge of an $\mathbf{a}+\mathbf{a}^{\prime}$ simple root to be from the hidden sector $S O(6)$. Thus, for model 2 the gauge boson from $\mathbf{a}+\mathbf{a}^{\prime}$ again regenerates a hidden sector $S O(10)$ :

$$
\left[S U(2)_{L}\right]^{O} \otimes\left[S O(6) \otimes S U(2)_{R}\right]^{H} \rightarrow[S O(10)]^{H} .
$$

However, in model 2, no additional gauge state is produced to mix the shadow sector with the hidden sector $S U(2)_{L}$. The final model 2 gauge group is, therefore,

$$
\left[S U(4) \otimes S U(2)_{R}\right]^{O} \otimes\left[S U(3) \times S U(2)^{3} \times U(1)^{7}\right]^{S} \otimes\left[S O(10) \otimes S U(2)_{L}\right]^{H} .
$$

(An exchange of definitions of left and right-handedness in the observable sector and of related SM states transforms this into

$$
\left.\left[S U(4) \otimes S U(2)_{L}\right]^{O} \otimes\left[S U(3) \times S U(2)^{3} \times U(1)^{7}\right]^{S} \otimes\left[S O(10) \otimes S U(2)_{L}\right]^{H} .\right)
$$




\section{Hypercharge Definitions}

An important issue for these models is whether an acceptable definition of hypercharge can be found, since the conventional hypercharge is missing either its equivalent $T_{3}^{R}$ contribution in model 1 or its equivalent $T_{3}^{L}$ contribution in model 2 . In standard NAHE-based models the hypercharge is formed as

$$
Y=\frac{1}{3} \tilde{Q}_{C}+\frac{1}{2} \tilde{Q}_{L}
$$

(for $Y\left(Q_{L}\right)=\frac{1}{3}$ normalization), where $\tilde{Q}_{C}=\sum_{m=1}^{3} Q_{\bar{\psi}^{m}}$ is the associated charge trace of $U(1)_{C} \equiv\left[\bar{\psi}^{1 *} \bar{\psi}^{1}+\bar{\psi}^{2 *} \bar{\psi}^{2}+\bar{\psi}^{3 *} \bar{\psi}^{3}\right]$ and $\tilde{Q}_{L}=\sum_{m=4}^{5} Q_{\bar{\psi}^{m}}$ is the associated charge trace of $U(1)_{L} \equiv\left[\bar{\psi}^{4 *} \bar{\psi}^{4}+\bar{\psi}^{5 *} \bar{\psi}^{5}\right]$. Since $S U(4)_{C} \rightarrow S U(3)_{C} \otimes U(1)_{B-L}, \frac{1}{3} \tilde{Q}_{C}=\tilde{Q}_{B-L}$, as Table 4.a indicates. Similarly, since $S O(4)=S U(2)_{L} \otimes S U(2)_{R} \rightarrow S U(2)_{L} \otimes U(1)_{L}$, $\frac{1}{2} \tilde{Q}_{L}=2 T_{3}^{R}$. Thus,

$$
Y=\tilde{Q}_{B-L}+2 T_{3}^{R}
$$

which yields electromagnetic charge

$$
\tilde{Q}_{E M}=T_{3}^{L}+\frac{1}{2} Y=T_{3}^{L}+T_{3}^{R}+\frac{1}{2} \tilde{Q}_{B-L} .
$$

Hence, model 1 requires a replacement for $T_{3}^{R}$, while model 2 needs a replacement for $T_{3}^{L}$.

Under $S U(4)_{C} \otimes S U(2)_{L}$ the $\mathrm{SM} S U(3)_{C} \otimes S U(2)_{L}$ left handed reps combine into

- $Q_{L}=(\mathbf{3}, \mathbf{2})_{2 T_{3}^{R}=0} \oplus L_{L}=(\mathbf{1}, \mathbf{2})_{2 T_{3}^{R}=0} \rightarrow(Q L)_{L}=(\mathbf{4}, \mathbf{2})_{2 T_{3}^{R}=0}$,

- $e_{L}^{c}=(\mathbf{1}, \mathbf{1})_{2 T_{3}^{R}=1} \oplus d_{L}^{c}=(\mathbf{3}, \mathbf{1})_{2 T_{3}^{R}=1} \rightarrow\left(d^{c} e^{c}\right)_{L}=(\mathbf{4}, \mathbf{1})_{2 T_{3}^{R}=1}$,

- $\nu_{L}^{c}=(\mathbf{1}, \mathbf{1})_{2 T_{3}^{R}=-1} \oplus u_{L}^{c}=(\mathbf{3}, \mathbf{1})_{2 T_{3}^{R}=-1} \rightarrow\left(u^{c} \nu^{c}\right)_{L}=(\mathbf{4}, \mathbf{1})_{2 T_{3}^{R}=-1}$.

Model 1 contains three generations of pairs of $\left(q^{c} l^{c}\right)_{i}^{n}=(\mathbf{4}, \mathbf{1})$ states (with generation index specified by $i=1$ to 3 , pair element index specified by $n=1,2$, and left-handed index L implicit), where $\left(q^{c} l^{c}\right)$ denotes either $\left(d^{c} e^{c}\right)$ or $\left(u^{c} \nu^{c}\right)$ (see Table B.3). These states are also respective doublets under the three generation $S U(2)_{i}$ of the shadow sector. As discussed, for a three generation model, the $S U(2)_{i}$ must be broken to the generational $U(1)_{i}$ of standard NAHE models by additional GSO projections from further sectors. When each $S U(2)_{i}$ is broken to $U(1)_{i}$ in this manner, one component of each $S U(2)_{i}$ doublet is also projected out. If these additional GSO projections can be chosen such that the up-spin component of $S U(2)_{i}$ for $\left(q^{c} l^{c}\right)_{i}^{n=1}$ survives along with the down-spin component of $S U(2)_{i}$ for $\left(q^{c} l^{c}\right)_{i}^{n=2}$, then for $Y=\tilde{Q}_{B-L}+2\left(T_{3}\right)_{i}$ the $\left(q^{c} l^{c}\right)_{i}^{n=1}$ become the $\left(d^{c} e^{c}\right)_{i}$ states and the $\left(q^{c} l^{c}\right)_{i}^{n=2}$ become the $\left(u^{c} \nu^{c}\right)_{i}$ states. Under $L \leftrightarrow R$ exchange, the same process can be applied to create a consistent three generation hypercharge for model 2 . 
For both models, this is the only possible choice for $\left(q^{c} l^{c}\right)$ hypercharge, since in each model the extra Abelian charges carried by the $\left(q^{c} l^{c}\right)_{i}^{n}$ are independent of the index $n$ for each generation $i$. That is, no hypercharge definition involving only the extra $U(1)_{k}$ could yield valid hypercharge for both $\left(d^{c} e^{c}\right)$ and $\left(u^{c} \nu^{c}\right)$ reps. For model 1 , this posses a difficulty for the MSSM higgs. In model 1 , the only additional $S U(2)_{L}$ doublets are singlets under all $S U(2)_{i}$. These are the pairs of states $h_{i}^{n}(n=1,2)$ and the more exotic $H_{i}^{n}$, which are also 5 reps of $S U(5)^{S}$. For a given generation $i$, the extra $U(1)_{k}$ charges are independent of the index $n$. Thus, no hypercharge definition could yield both a $(Y=-1)$-charged up-higgs and a $(Y=+1)$-charged down-higgs from an $h_{i}^{n=1}$ and $h_{i}^{n=2}$ pair. Instead, a hypercharge definition is required such that the $U(1)_{k}$ hypercharge contribution to $Q L$ and $q l$ states is zero, while it is +1 for at least one $h_{i}^{n}$ pair and -1 for at least one other $h_{i^{\prime}}^{n}$ pair. Applying the six $Q L$ and $\left(q^{c} l^{c}\right)$ constraints prevents any $U(1)_{k}$ from appearing in a general hypercharge definition. Thus, model 1 cannot provide a suitable definition for hypercharge, unless the Cartan subalgebra of $S U(5)^{S}$ can contribute to the hypercharge of $H_{i}^{n}$ components after $S U(5)^{S}$ is broken.

In contrast, the MSSM higgs of model 2 come in the standard $h_{i}$ and $\bar{h}_{i}$ pairs for each generation i. However, each $h_{i}$ and $\bar{h}_{i}$ is also an $S U(2)_{i}$ doublet (see Table C.3). Thus, if under $S U(2)_{i}$ breaking by GSO projections from additional sectors, the $S U(2)_{i}$ up-spin component of $h_{i}$ survives and the $S U(2)_{i}$ down-spin component of $\bar{h}_{i}$ (or vice-versa) then $h_{i}$ becomes down-higgs and $\bar{h}_{i}$ becomes up-higgs (or vice versa). Thus, a viable hypercharge definition for model 2 is

$$
Y=\frac{1}{2} \tilde{Q}_{B-L}+\sum_{i=1}^{3} T_{3}^{i}
$$

This would produce generational higgs pairs, which is a common occurrence in NAHE-based models. This often provides for mass hierarchy between generations since the physical higgs usually becomes a weighted (by several orders of magnitude) linear combination of the generational higgs. MSSM matter states then couple differently by generation to the physical higgs, producing a large mass hierarchy, even when all mass couplings in the superpotential are third order.

Models 1 and 2 both contain an anomalous $U(1)$ and it is unlikely that additional basis vectors would remove the anomaly from either model [19]. In fact, additional sectors generally increase the anomaly. For model 2 the charge traces of the seven $U(1)_{k}$ are

$$
\operatorname{Tr} \mathbf{Q}=(0,-144,96,0,0,-192,0) .
$$

(as can be computed from Table C.3). The anomaly may be rotated into a single $U(1)_{A}$,

$$
U(1)_{A}=\left[-3 Q_{2}+2 Q_{3}-4 Q_{6}\right]
$$


for which the trace is 1392 . The orthogonal

$$
\begin{aligned}
& U_{2}^{\prime}=2 Q_{2}+3 Q_{3} \\
& U_{3}^{\prime}=-3 Q_{2}+2 Q_{3}+(13 / 4) Q_{6} .
\end{aligned}
$$

become non-anomalous (traceless).

Model 2 is another example [12] for which non-Abelian fields must necessarily take on VEVs to cancel the Fayet-Iliopoulos (FI) term,

$$
\epsilon \equiv \frac{g_{s}^{2} M_{P}^{2}}{192 \pi^{2}} \operatorname{Tr} Q^{(A)}=\frac{g_{s}^{2} M_{P}^{2}}{192 \pi^{2}} 1392,
$$

generated in the $U_{A} D$-term by the Green-Schwarz-Dine-Seiberg-Witten anomalous U(1) breaking mechanism [20]. To see this, first note from Table C.3 that singlet states $S_{1}^{n}$ through $S_{6}^{n}$ carry the non-anomalous charge $Q_{1}=3$, while the remaining singlets have $Q_{1}=0$. Thus, $D$-flatness for $U(1)_{1}$ cannot be maintained if only singlets receive VEVs and one or more of the fields $S_{1}^{i}$ through $S_{6}^{i}$ are among those that do. Next, the singlets $S_{7}$ and $\bar{S}_{7}$ do not carry anomalous charge (only $Q_{4}$ and $Q_{5}$ charge), and so cannot help cancel the F-I term. The remaining singlets are simply $S_{8}$ and $S_{9}$, and their vector partners of opposite charges. $S_{8}$ carries anomalous charge $Q_{A}=4$ and non-zero non-anomalous charges $Q_{4}=1, Q_{5}=4, Q_{2}^{\prime}=6, Q_{3}^{\prime}=-42$, while $S_{9}$ carries anomalous charge $Q_{A}=4$ and non-zero non-anomalous charges $Q_{4}=-1$, $Q_{5}=-4, Q_{2}^{\prime}=6, Q_{3}^{\prime}=-42 . \bar{S}_{8}$ and $\bar{S}_{9}$ carry respective opposite charges. Thus, we see that no combination of $S_{7,8,9}$ and $\bar{S}_{7,8,9}$ VEVs can simultaneously cancel the anomalous $D_{A}$-term contribution from the trace of $U_{A}$ and keep the D-terms for $Q_{2}^{\prime}$ and $Q_{3}^{\prime}$ flat. Therefore, some non-Abelian fields must take on VEVs in the process of cancelling the $\operatorname{Tr} Q_{A}$ contribution to $D_{A}$ to maintain $D$-flatness. Of particular interest is whether $S U(2)_{i}$-charged fields take on VEVs in the parameter space of flat directions. Analysis of flat directions for these models is, however, beyond the scope of this letter.

\section{Summary}

We have demonstrated how, under certain conditions, mirror symmetry is necessarily broken between the observable and hidden sector gauge groups of heterotic string models with mirror boundary conditions for observable and hidden sector worldsheet fermions. The observable/hidden sector gauge group mirror breaking occurs because of an unavoidable asymmetry in GSO projections. This effect can be induced in free fermionic models through an observable/hidden sector mirror pair of basis vectors, a and $\mathbf{a}^{\prime}$, with the properties that:

- Their vector sum yields new, independent gauge sectors (possibly after further basis vectors are added) $\mathbf{a}+\mathbf{a}^{\prime}+\ldots$ 
- They do not overlap with non-zero components in the observable and hidden sectors.

- Their inner product is not equal $0(\bmod 4)$.

Under these conditions the observable and hidden sector gauge states from some $\mathbf{a}+\mathbf{a}^{\prime}+\ldots$ sector (or sectors) will not be mirror images, since the observable gauge states surviving the $k_{\mathbf{a}, \mathbf{a}+\mathbf{a}^{\prime}+\ldots}$ GSO projections will be different from the hidden sector gauge states surviving the corresponding $k_{\mathbf{a}^{\prime}, \mathbf{a}+\mathbf{a}^{\prime}+\ldots}$ GSO projections. In the examples shown, starting with mirror Pati-Salam gauge groups $\left[S U(4)_{C} \otimes S U(2)_{L} \otimes S U(2)_{R}\right]^{O} \otimes$ $\left[S U(4)_{C} \otimes S U(2)_{L} \otimes S U(2)_{R}\right]^{H}$, the observable sector $S U(2)_{R(L)}$ was transformed to the hidden sector by this necessary asymmetry of the GSO projections, enhancing the hidden sector gauge group to $\left[S O(10) \otimes S U(2)_{R(L)}\right]^{H}$. This transference of gauge rank from the observable sector to the hidden sector acts favorably for coupling strength renormalizations, allowing non-Abelian hidden sector coupling strengths to increase faster than observable sector coupling strengths, with decreasing energy below the string scale, leading to the formation of generally advantageous intermediate scale hidden sector condensates.

\section{Acknowledgments}

This work is supported in part by the NASA/Texas Space Grant Consortium. 


\section{References}

[1] Z. Berezhiani, A. Dolgov, and R. Mohapatra, Phys. Lett. B375 (1996) 26-36, [arXiv: hep-ph/9511221].

[2] R. Mohapatra and V. Teplitz, Astrophys. Jour. 478 (1997) 29-38, [arXiv: astro$\mathrm{ph} / 9603049]$.

[3] Z. Berezhiani, D. Comelli, and F. Villante, Phys. Lett. B5053 (2001) 362-375, [arXiv: hep-ph/0008105].

[4] Z. Silagadze, Acta. Phys. Polon. B32 (2001) 99-128, [arXiv: hep-ph/0002255].

[5] P. Ciarcelluti, Thesis, [astro-ph/0312607].

[6] B. Dundee, J. Perkins, G. Cleaver, research in progress.

[7] J. Pati and A. Salam, Phys. Rev. D10 (1974) 275; J. Pati, Phys. Rev. Lett. 31 (1973) 661; R. Mohapatra and J. Pati, Phys. Rev. D11 (1975) 566; G. Senjanovic and R. Mohapatra, Phys. Rev. D12 (1975) 1502.

[8] I. Antoniadis, G. Leontaris, and J. Rizos, Phys. Lett. B245 (1990) 161; G. Leontaris and J. Rizos, Nucl. Phys. B554 (1999) 3.

[9] I. Antoniadis, C. Bachas, and C. Kounnas, Nucl. Phys. B289 (1987) 87; H. Kawai, D.C. Lewellen, and S.H.-H. Tye, Nucl. Phys. B288 (1987) 1.

[10] A. Faraggi and D. Nanopoulos, Phys. Rev. D48 (1993) 3288; A. Faraggi, [hepth/9511093]; [hep-th/9708112].

[11] I. Antoniadis, J. Ellis, J. Hagelin, and D. Nanopoulos, Phys. Lett. B231 (1989) 65; J. Lopez and D. Nanopoulos, Phys. Lett. B268 (1991) 359; J. Ellis, J. Lopez, D. Nanopoulos, and K. Olive, Phys. Lett. B308 (1993) 70, [hep-ph/9303307]; J. Lopez and D. Nanopoulos, [hep-ph/9511266]; J. Lopez and D. Nanopoulos, Phys. Rev. D55 (1997) 397, [hep-ph/9605359]; J. Lopez and D. V. Nanopoulos, [hep-ph/9701264]; J. Ellis, G. Leontaris, S. Lola, and D. Nanopoulos, Phys. Lett. B425 (1998) 86, [hep-ph/9711476]; G. Cleaver, J. Ellis, and D. Nanopoulos, Nucl. Phys. B600 (2001) 315, [hep-ph/0009338]; J. Ellis, D. Nanopoulos, and J. Walker, Phys. Lett. B550 (2002) 99, [hep-ph/0205336]; D. Nanopoulos, [hepph/0211128]; J. Ellis, V. Mayes, and D. Nanopoulos, Phys. Rev. D70 (2004) 075015, [hep-ph/0403144].

[12] G. Cleaver, A. Faraggi, and C. Savage, Phys. Rev. D63 (2001) 066001, [hepph/0006331]; G. Cleaver, D. Clements, and A. Faraggi, Phys. Rev. D65 (2002) 106003, [hep-ph/0106060]; G. Cleaver, A. Faraggi, and S. Nooij, Nucl. Phys. B672 (2003) 64, [hep-ph/0301037]. 
[13] S. Kelley, J. Lopez, and D. Nanopoulos, Phys. Lett. B278 (1992) 140; A. Faraggi, D. Nanopoulos, and K. Yuan, Nucl. Phys. B335 (1990) 347; A. Faraggi, Phys. Rev. D46 (1992) 3204; Phys. Lett. B278 (1992) 131; Nucl. Phys. B403 (1992) 101; Phys. Lett. B274 (1992) 47; Phys. Lett. B339 (1994) 223; Phys. Lett. B302 (1993) 202; Phys. Lett. B339 (1994) 223; Nucl. Phys. BB387 (1992) 239.

[14] G. Cleaver, V. Desai, H. Hanson, J. Perkins, D. Robbins, and S. Shields, Phys. Rev. D67 (2003) 026009, [hep-ph/0209050]; J. Perkins, B. Dundee, R. Obousy, E. Kasper, M. Robinson, K. Stone, and G. Cleaver, [hep-ph/0310155].

[15] G. Cleaver, A. Faraggi, and D. Nanopoulos, Phys. Lett. B455 (1999) 135, [hepph/9811427]; Int. J. Mod. Phys. A16 (2001) 425, [hep-ph/9904301]; G. Cleaver, A. Faraggi, D. Nanopoulos, and J. Walker, NPB5932001471, [hep-ph/9910230]; Mod. Phys. Lett. A15 (2000) 1191, [hep-ph/0002060]; Nucl. Phys. B620 (2002) 259, [hep-ph/0104091]; G. Cleaver, Int. J. Mod. Phys. A16S1C (2001) 949, [hep-ph/0011020]; [hep-ph/0003208].

[16] S. Kalara, J. Lopez, and D. Nanopoulos, Phys. Lett. B245 (1990) 421; A. Faraggi, and D. Nanopoulos, Phys. Rev. D48 (1993) 3288; I. Giannakis, D. Nanopoulos, and K. Yuan, Phys. Rev. D52 (1995) 1026, [hep-th/9502153]; G. Cleaver, A. Faraggi, D. Nanopoulos, and T. ter Veldhuis, Int. J. Mod. Phys. A16 (2001) 3565, [hep-ph/0002292]; G. Cleaver, [hep-ph/0210093]; R. Donagi, and A. Faraggi, Nucl. Phys. B694 (2004) 187, [hep-th/0403272]; A. Faraggi, C. Kounnas, S. Nooij, and J. Rizos, [hep-th/0311058]; A. Faraggi, C. Kounnas, S. Nooij, and J. Rizos, Nucl. Phys. B695 (2004) 41, [hep-th/0403058].

[17] A. Faraggi, R. Garavuso, and J. Isidro, Nucl. Phys. B641 (2002) 111; [hepth/0209245]; A. Faraggi, Int. J. Mod. Phys. A19 (2004) 5523, [hep-th/0411118]; [hep-th/0307037]; D. Clements and A. Faraggi, Int. J. Mod. Phys. A19 (2004) 2931, [hep-th/0302006].

[18] A. Faraggi and J. Pati, Nucl. Phys. B526 (1998) 21; A. Faraggi, [hepth/0504016].

[19] G. Cleaver and A. Faraggi, Int. J. Mod. Phys. A14 (1999) 2335; A. Faraggi, Phys. Lett. B246 (1998) 315, [hep-ph/9801409]; A. Faraggi, Nucl. Phys. B641 (2002) 93, [hep-ph/0203061].

[20] M. Green and J. Schwarz, Phys. Lett. B149 (1984) 117;

M. Dine, N. Seiberg, and E. Witten, Nucl. Phys. B289 (1987) 589;

J. Atick, L. Dixon, and A. Sen, Nucl. Phys. B292 (1987) 109. 
Table A.1 THE NAHE SET

\begin{tabular}{|c|c|ccc|c|ccc|c|ccc|}
\hline & $\psi^{\mu}$ & $\chi^{12}$ & $\chi^{34}$ & $\chi^{56}$ & $\bar{\psi}^{1, \ldots, 5}$ & $\bar{\eta}^{1}$ & $\bar{\eta}^{2}$ & $\bar{\eta}^{3}$ & $\bar{\psi}^{1, \ldots, 5}$ & ${\overline{\eta^{\prime}}}^{1}$ & ${\overline{\eta^{\prime}}}^{2}$ & $\bar{\eta}^{3}$ \\
\hline \hline $\mathbf{1}$ & 1 & 1 & 1 & 1 & $1, \ldots, 1$ & 1 & 1 & 1 & $1, \ldots, 1$ & 1 & 1 & 1 \\
$\mathbf{S}$ & 1 & 1 & 1 & 1 & $0, \ldots, 0$ & 0 & 0 & 0 & $0, \ldots, 0$ & 0 & 0 & 0 \\
\hline $\mathbf{b}_{1}$ & 1 & 1 & 0 & 0 & $1, \ldots, 1$ & 1 & 0 & 0 & $0, \ldots, 0$ & 0 & 0 & 0 \\
$\mathbf{b}_{2}$ & 1 & 0 & 1 & 0 & $1, \ldots, 1$ & 0 & 1 & 0 & $0, \ldots, 0$ & 0 & 0 & 0 \\
$\mathbf{b}_{3}$ & 1 & 0 & 0 & 1 & $1, \ldots, 1$ & 0 & 0 & 1 & $0, \ldots, 0$ & 0 & 0 & 0 \\
\hline
\end{tabular}

\begin{tabular}{|c|cc|cc|cc|}
\hline & $y^{3, \ldots, 6}$ & $\bar{y}^{3, \ldots, 6}$ & $y^{1,2}, \omega^{5,6}$ & $\bar{y}^{1,2}, \bar{\omega}^{5,6}$ & $\omega^{1, \ldots, 4}$ & $\bar{\omega}^{1, \ldots, 4}$ \\
\hline \hline $\mathbf{1}$ & $1, \ldots, 1$ & $1, \ldots, 1$ & $1, \ldots, 1$ & $1, \ldots, 1$ & $1, \ldots, 1$ & $1, \ldots, 1$ \\
$\mathbf{S}$ & $0, \ldots, 0$ & $0, \ldots, 0$ & $0, \ldots, 0$ & $0, \ldots, 0$ & $0, \ldots, 0$ & $0, \ldots, 0$ \\
\hline $\mathbf{b}_{1}$ & $1, \ldots, 1$ & $1, \ldots, 1$ & $0, \ldots, 0$ & $0, \ldots, 0$ & $0, \ldots, 0$ & $0, \ldots, 0$ \\
$\mathbf{b}_{2}$ & $0, \ldots, 0$ & $0, \ldots, 0$ & $1, \ldots, 1$ & $1, \ldots, 1$ & $0, \ldots, 0$ & $0, \ldots, 0$ \\
$\mathbf{b}_{3}$ & $0, \ldots, 0$ & $0, \ldots, 0$ & $0, \ldots, 0$ & $0, \ldots, 0$ & $1, \ldots, 1$ & $1, \ldots, 1$ \\
\hline
\end{tabular}

Table A.2 Mirror Set

\begin{tabular}{|c|c|ccc|c|ccc|c|ccc|}
\hline & $\psi^{\mu}$ & $\chi^{12}$ & $\chi^{34}$ & $\chi^{56}$ & $\bar{\psi}^{1, \ldots, 5}$ & $\bar{\eta}^{1}$ & $\bar{\eta}^{2}$ & $\bar{\eta}^{3}$ & $\bar{\psi}^{1, \ldots, 5}$ & $\bar{\eta}^{\prime 1}$ & $\bar{\eta}^{\prime 2}$ & $\bar{\eta}^{\prime}$ \\
\hline \hline $\mathbf{b}_{1}^{\prime}$ & 1 & 1 & 0 & 0 & $0, \ldots, 0$ & 0 & 0 & 0 & $1, \ldots, 1$ & 1 & 0 & 0 \\
$\mathbf{b}_{2}^{\prime}$ & 1 & 0 & 1 & 0 & $0, \ldots, 0$ & 0 & 0 & 0 & $1, \ldots, 1$ & 0 & 1 & 0 \\
$\mathbf{b}_{3}^{\prime}$ & 1 & 0 & 0 & 1 & $0, \ldots, 0$ & 0 & 0 & 0 & $1, \ldots, 1$ & 0 & 0 & 1 \\
\hline
\end{tabular}

\begin{tabular}{|c|cc|cc|cc|}
\hline & $y^{1, \ldots, 4}$ & $\bar{y}^{1, \ldots, 4}$ & $y^{5,6}, \omega^{1,2}$ & $\bar{y}^{5,6}, \bar{\omega}^{1,2}$ & $\omega^{3, \ldots, 6}$ & $\bar{\omega}^{3, \ldots, 6}$ \\
\hline \hline $\mathbf{b}^{\prime}{ }_{1}$ & $1, \ldots, 1$ & $1, \ldots, 1$ & $0, \ldots, 0$ & $0, \ldots, 0$ & $0, \ldots, 0$ & $0, \ldots, 0$ \\
$\mathbf{b}^{\prime}{ }_{2}$ & $0, \ldots, 0$ & $0, \ldots, 0$ & $1, \ldots, 1$ & $1, \ldots, 1$ & $0, \ldots, 0$ & $0, \ldots, 0$ \\
$\mathbf{b}^{\prime}{ }_{3}$ & $0, \ldots, 0$ & $0, \ldots, 0$ & $0, \ldots, 0$ & $0, \ldots, 0$ & $1, \ldots, 1$ & $1, \ldots, 1$ \\
\hline
\end{tabular}

Table A.3 $S O(10) \otimes S O(10)$ Breaking and Generation Reduction

\begin{tabular}{|c|c|ccc|c|ccc|c|c|ccc|}
\hline & $\psi^{\mu}$ & $\chi^{12}$ & $\chi^{34}$ & $\chi^{56}$ & $\bar{\psi}^{1, \ldots, 5}$ & $\bar{\eta}^{1}$ & $\bar{\eta}^{2}$ & $\bar{\eta}^{3}$ & $y, \bar{y}, \omega, \omega$ & $\bar{\psi}^{\prime 1, \ldots, 5}$ & ${\overline{\eta^{\prime}}}^{1}$ & ${\overline{\eta^{\prime}}}^{2}$ & ${\overline{\eta^{\prime}}}^{3}$ \\
\hline \hline $\mathbf{a}$ & 1 & 1 & 1 & 1 & $0,0,0,1,1$ & 1 & 1 & 1 & $\overrightarrow{0}$ & $0,0,0,0,0$ & 0 & 0 & 0 \\
$\mathbf{a}^{\prime}$ & 1 & 1 & 1 & 1 & $0,0,0,0,0$ & 0 & 0 & 0 & $\overrightarrow{0}$ & $0,0,0,1,1$ & 0 & 0 & 0 \\
\hline
\end{tabular}


Table B.1 Model 1 GSO Matrix k

\begin{tabular}{|l|rr|rrr|rrr|rr|}
\hline$k_{i, j}$ & $\mathbf{1}$ & $\mathbf{S}$ & $\mathbf{b}_{1}$ & $\mathbf{b}_{2}$ & $\mathbf{b}_{3}$ & $\mathbf{b}_{1}^{\prime}$ & $\mathbf{b}_{2}^{\prime}$ & $\mathbf{b}_{3}^{\prime}$ & $\mathbf{a}$ & $\mathbf{a}^{\prime}$ \\
\hline $\mathbf{1}$ & 0 & 0 & 1 & 1 & 1 & 1 & 1 & 1 & 0 & 0 \\
$\mathbf{S}$ & 0 & 0 & 0 & 0 & 0 & 0 & 0 & 0 & 0 & 0 \\
\hline $\mathbf{b}_{1}$ & 1 & 1 & 1 & 1 & 1 & 0 & 0 & 0 & 0 & 1 \\
$\mathbf{b}_{2}$ & 1 & 1 & 1 & 1 & 1 & 0 & 0 & 0 & 0 & 1 \\
$\mathbf{b}_{3}$ & 1 & 1 & 1 & 1 & 1 & 0 & 0 & 0 & 0 & 1 \\
\hline $\mathbf{b}_{1}^{\prime}$ & 1 & 1 & 0 & 0 & 0 & 1 & 1 & 1 & 1 & 0 \\
$\mathbf{b}_{2}^{\prime}$ & 1 & 1 & 0 & 0 & 0 & 1 & 1 & 1 & 1 & 0 \\
$\mathbf{b}_{3}^{\prime}$ & 1 & 1 & 0 & 0 & 0 & 1 & 1 & 1 & 1 & 0 \\
\hline $\mathbf{a}$ & 0 & 0 & 0 & 0 & 0 & 0 & 0 & 0 & 1 & ${ }^{*} 1$ \\
$\mathbf{a}^{\prime}$ & 0 & 0 & 0 & 0 & 0 & 0 & 0 & 0 & $* 0$ & 1 \\
\hline
\end{tabular}

Table B.2 Model 1 Gauge Group

\begin{tabular}{|l|rrrrr|}
\hline Observable & $\psi^{1}$ & $\psi^{2}$ & $\psi^{3}$ & $\psi^{3}$ & $\psi^{5}$ \\
\hline \hline$S U(4)_{C}$ & 0 & 1 & -1 & 0 & 0 \\
& 1 & 0 & -1 & 0 & 0 \\
& 0 & 1 & 1 & 0 & 0 \\
\hline$S U(2)_{L}$ & 0 & 0 & 0 & 1 & -1 \\
\hline
\end{tabular}

\begin{tabular}{|l|rrr|rrrrrr|rr|rrr|}
\hline Shadow & $\bar{\eta}^{1}$ & $\bar{\eta}^{2}$ & $\bar{\eta}^{3}$ & $\bar{y}_{1,3}$ & $\bar{y}_{2,4}$ & $\bar{y}_{5} \bar{w}_{1}$ & $\bar{y}_{6} \bar{\omega}_{2}$ & $\bar{\omega}_{3,5}$ & $\bar{\omega}_{4,6}$ & $\bar{\psi}^{\prime^{4}}$ & $\bar{\psi}^{\prime}$ & $\bar{\eta}^{\prime 1}$ & $\bar{\eta}^{\prime^{2}}$ & $\bar{\eta}^{3}$ \\
\hline \hline$S U(2)^{3}$ & $\frac{1}{2}$ & $-\frac{1}{2}$ & 0 & 0 & $\frac{1}{2}$ & 0 & $-\frac{1}{2}$ & $-\frac{1}{2}$ & $\frac{1}{2}$ & 0 & 0 & $-\frac{1}{2}$ & $\frac{1}{2}$ & 0 \\
& $\frac{1}{2}$ & 0 & $-\frac{1}{2}$ & $\frac{1}{2}$ & 0 & $-\frac{1}{2}$ & $\frac{1}{2}$ & 0 & $-\frac{1}{2}$ & 0 & 0 & $-\frac{1}{2}$ & 0 & $\frac{1}{2}$ \\
& 0 & $\frac{1}{2}$ & $-\frac{1}{2}$ & $-\frac{1}{2}$ & $\frac{1}{2}$ & $\frac{1}{2}$ & 0 & $-\frac{1}{2}$ & 0 & 0 & 0 & 0 & $-\frac{1}{2}$ & $\frac{1}{2}$ \\
\hline$S U(5)$ & 0 & 0 & 0 & 0 & 0 & 0 & 0 & 0 & 0 & 1 & 1 & 0 & 0 & 0 \\
& 1 & $-\frac{1}{2}$ & 0 & 0 & $-\frac{1}{2}$ & 0 & $\frac{1}{2}$ & $-\frac{1}{2}$ & $\frac{1}{2}$ & 0 & 0 & $\frac{1}{2}$ & $-\frac{1}{2}$ & 0 \\
& 0 & $\frac{1}{2}$ & $\frac{1}{2}$ & 0 & 0 & 0 & $-\frac{1}{2}$ & 0 & $-\frac{1}{2}$ & $\frac{1}{2}$ & $\frac{1}{2}$ & 0 & $\frac{1}{2}$ & $\frac{1}{2}$ \\
& 0 & $\frac{1}{2}$ & $-\frac{1}{2}$ & $-\frac{1}{2}$ & $\frac{1}{2}$ & $-\frac{1}{2}$ & 0 & $\frac{1}{2}$ & 0 & 0 & 0 & 0 & $\frac{1}{2}$ & $-\frac{1}{2}$ \\
\hline
\end{tabular}

\begin{tabular}{|c|rrrrr|rrrrr|}
\hline Hidden & $\bar{\psi}^{1}$ & $\bar{\psi}^{2}$ & $\bar{\psi}^{3}$ & $\bar{\psi}^{3}$ & $\bar{\psi}^{5}$ & $\bar{\psi}^{1}$ & $\bar{\psi}^{2}$ & $\bar{\psi}^{3}$ & ${\overline{\psi^{3}}}^{3}$ & $\bar{\psi}^{5}$ \\
\hline \hline$S O(10)$ & 0 & 0 & 0 & 0 & 0 & 0 & 0 & 0 & 1 & -1 \\
& 0 & 0 & 0 & $\frac{1}{2}$ & $\frac{1}{2}$ & 0 & 0 & -1 & $\frac{1}{2}$ & $-\frac{1}{2}$ \\
& 0 & 0 & 0 & 0 & 0 & 0 & 1 & -1 & 0 & 0 \\
& 0 & 0 & 0 & 0 & 0 & 1 & -1 & 0 & 0 & 0 \\
& 0 & 0 & 0 & 0 & 0 & 1 & 1 & 0 & 0 & 0 \\
\hline
\end{tabular}


Table B.3 Model 1 Left-Handed States

\begin{tabular}{|l|rlr|rrrrrr|}
\hline$n=1,2$ & $\left(4_{C}, 2_{L}\right)_{O}$ & $\left(2^{3}, 5\right)_{S}$ & $10_{H}$ & $4 Q_{1}$ & $4 Q_{2}$ & $4 Q_{3}$ & $4 Q_{4}$ & $4 Q_{5}$ & $4 Q_{6}$ \\
\hline Singlets & & & & & & & & & \\
\hline$S_{1}$ & $(1,1)$ & $(1,1,1,1)$ & $(1)$ & 0 & -12 & -4 & -36 & 128 & 80 \\
$\bar{S}_{1}$ & $(1,1)$ & $(1,1,1,1)$ & $(1)$ & 0 & 12 & 4 & 36 & -128 & -80 \\
$S_{2}$ & $(1,1)$ & $(1,1,1,1)$ & $(1)$ & -4 & 4 & 4 & -4 & 176 & 0 \\
$\bar{S}_{2}$ & $(1,1)$ & $(1,1,1,1)$ & $(1)$ & 4 & -4 & -4 & 4 & -176 & 0 \\
$S_{3}$ & $(1,1)$ & $(1,1,1,1)$ & $(1)$ & -4 & 16 & 8 & 32 & 48 & -80 \\
$\bar{S}_{3}$ & $(1,1)$ & $(1,1,1,1)$ & $(1)$ & 4 & -16 & -8 & -32 & -48 & 80 \\
\hline Observable & & & & & & & & & \\
\hline$Q L_{1}^{n}$ & $(4,2)$ & $(1,1,1,1)$ & $(1)$ & 0 & -6 & -6 & 6 & -40 & -80 \\
$Q L_{2}^{n}$ & $(4,2)$ & $(1,1,1,1)$ & $(1)$ & 0 & 0 & -4 & -56 & 0 & 0 \\
$Q L_{3}^{n}$ & $(4,2)$ & $(1,1,1,1)$ & $(1)$ & -6 & 0 & 0 & 0 & 0 & 0 \\
\hline$\left(q^{c} l^{c}\right)_{1}^{n}$ & $(-4,1)$ & $(2,1,1,1)$ & $(1)$ & -2 & -4 & 12 & -12 & 24 & -40 \\
$\left(q^{c} l^{c}\right)_{2}^{n}$ & $(-4,1)$ & $(1,2,1,1)$ & $(1)$ & 0 & -12 & 8 & -28 & 0 & 0 \\
$\left(q^{c} l^{c}\right)_{3}^{n}$ & $(-4,1)$ & $(1,1,2,1)$ & $(1)$ & 0 & -6 & 10 & -10 & -64 & -40 \\
\hline$h_{1}^{n}$ & $(1,2)$ & $(1,1,1,1)$ & $(1)$ & 0 & -6 & -10 & -50 & -40 & -80 \\
$h_{2}^{n}$ & $(1,2)$ & $(1,1,1,1)$ & $(1)$ & -6 & -6 & -6 & 6 & -40 & -80 \\
$h_{3}^{n}$ & $(1,2)$ & $(1,1,1,1)$ & $(1)$ & -6 & 0 & -4 & -56 & 0 & 0 \\
\hline$H_{1}^{n}$ & $(1,2)$ & $(1,1,1,5)$ & $(1)$ & 2 & -8 & 4 & 16 & 80 & -16 \\
$H_{2}^{n}$ & $(1,2)$ & $(1,1,1,5)$ & $(1)$ & 2 & 10 & 10 & -10 & -8 & -16 \\
$H_{3}^{n}$ & $(1,2)$ & $(1,1,1,5)$ & $(1)$ & 0 & -6 & 6 & 14 & -56 & 64 \\
\hline
\end{tabular}


Table B.3 Model 1 Left-Handed States, cont.

\begin{tabular}{|l|rlr|rrrrrr|}
\hline$n=1,2$ & $\left(4_{C}, 2_{L}\right)_{O}$ & $\left(2^{3}, 5\right)_{S}$ & $10_{H}$ & $4 Q_{1}$ & $4 Q_{2}$ & $4 Q_{3}$ & $4 Q_{4}$ & $4 Q_{5}$ & $4 Q_{6}$ \\
\hline Shadow & & & & & & & & & \\
\hline$F_{1}$ & $(1,1)$ & $(1,1,1,5)$ & $(1)$ & 0 & -12 & -4 & -36 & -96 & -16 \\
$\bar{F}_{1}$ & $(1,1)$ & $(1,1,1, \overline{5})$ & $(1)$ & 0 & 12 & 4 & 36 & 96 & 16 \\
$F_{2}$ & $(1,1)$ & $(1,1,1,5)$ & $(1)$ & -4 & -8 & 0 & -40 & 80 & -16 \\
$\bar{F}_{2}$ & $(1,1)$ & $(1,1,1, \overline{5})$ & $(1)$ & 4 & 8 & 0 & 40 & -80 & 16 \\
$F_{3}$ & $(1,1)$ & $(1,1,1,5)$ & $(1)$ & -4 & 4 & 4 & -4 & -48 & -96 \\
$\bar{F}_{3}$ & $(1,1)$ & $(1,1,1, \overline{5})$ & $(1)$ & 4 & -4 & -4 & 4 & 48 & 96 \\
\hline $\bar{G}_{1}^{n}$ & $(1,1)$ & $(2,1,1,10)$ & $(1)$ & 0 & -6 & -2 & -18 & -48 & -8 \\
$\bar{G}_{2}^{n}$ & $(1,1)$ & $(1,2,1, \overline{10})$ & $(1)$ & -2 & 2 & 2 & -2 & -24 & -48 \\
$\bar{G}_{3}^{n}$ & $(1,1)$ & $(1,1,2, \overline{10})$ & $(1)$ & -2 & -4 & 0 & -20 & 40 & -8 \\
\hline$X_{1}^{n}$ & $(1,1)$ & $(2,1,1,1)$ & $(1)$ & -2 & -4 & 16 & 44 & 24 & -40 \\
$X_{2}^{n}$ & $(1,1)$ & $(2,1,1,1)$ & $(1)$ & -2 & 2 & 18 & -18 & 64 & 40 \\
$X_{3}^{n}$ & $(1,1)$ & $(2,1,1,1)$ & $(1)$ & -2 & 2 & -14 & 14 & 112 & -40 \\
$X_{4}^{n}$ & $(1,1)$ & $(2,1,1,1)$ & $(1)$ & -2 & 20 & -8 & -12 & 24 & -40 \\
\hline$Y_{1}^{n}$ & $(1,1)$ & $(1,2,1,1)$ & $(1)$ & 0 & -6 & -18 & -2 & 88 & 0 \\
$Y_{2}^{n}$ & $(1,1)$ & $(1,2,1,1)$ & $(1)$ & 6 & -12 & 8 & -28 & 0 & 0 \\
$Y_{3}^{n}$ & $(1,1)$ & $(1,2,1,1)$ & $(1)$ & 0 & -6 & 14 & -34 & 40 & 80 \\
$Y_{4}^{n}$ & $(1,1)$ & $(1,2,1,1)$ & $(1)$ & -2 & -4 & -16 & -4 & -48 & 80 \\
\hline$Z_{1}^{n}$ & $(1,1)$ & $(1,1,2,1)$ & $(1)$ & -2 & 2 & -14 & 14 & -112 & 40 \\
$Z_{2}^{n}$ & $(1,1)$ & $(1,1,2,1)$ & $(1)$ & 0 & -6 & 14 & 46 & -64 & -40 \\
$Z_{3}^{n}$ & $(1,1)$ & $(1,1,2,1)$ & $(1)$ & 0 & 18 & -10 & -10 & -64 & -40 \\
$Z_{4}^{n}$ & $(1,1)$ & $(1,1,2,1)$ & $(1)$ & 6 & -6 & 10 & -10 & -64 & -40 \\
\hline$U_{1}$ & $(1,1)$ & $(2,2,1,1)$ & $(1)$ & -4 & -8 & 0 & 40 & -24 & 40 \\
$\bar{U}_{1}$ & $(1,1)$ & $(2,2,1,1)$ & $(1)$ & 4 & 8 & 0 & -40 & 24 & -40 \\
$U_{2}$ & $(1,1)$ & $(2,1,2,1)$ & $(1)$ & -4 & 4 & 4 & -4 & -48 & 80 \\
$\bar{U}_{2}$ & $(1,1)$ & $(2,1,2,1)$ & $(1)$ & 4 & -4 & -4 & 4 & 48 & -80 \\
$U_{3}$ & $(1,1)$ & $(1,2,2,1)$ & $(1)$ & 0 & -12 & -4 & 44 & 24 & -40 \\
$\bar{U}_{3}$ & $(1,1)$ & $(1,2,2,1)$ & $(1)$ & 0 & 12 & 4 & -44 & -24 & 40 \\
\hline Hidden & & & & & & & & & \\
\hline$T_{1}^{n}$ & $(1,1)$ & $(1,1,1,1)$ & $(16)$ & -2 & -16 & 0 & 0 & 0 & 0 \\
$T_{2}^{n}$ & $(1,1)$ & $(1,1,1,1)$ & $(16)$ & -2 & 2 & 6 & -26 & -88 & 0 \\
$T_{3}^{n}$ & $(1,1)$ & $(1,1,1,1)$ & $(16)$ & 0 & 0 & 4 & -24 & -24 & 0 \\
\hline
\end{tabular}


Table C.1 Model 2 GSO Matrix k

\begin{tabular}{|l|rr|rrr|rrr|rr|}
\hline$k_{i, j}$ & $\mathbf{1}$ & $\mathbf{S}$ & $\mathbf{b}_{1}$ & $\mathbf{b}_{2}$ & $\mathbf{b}_{3}$ & $\mathbf{b}_{1}^{\prime}$ & $\mathbf{b}_{2}^{\prime}$ & $\mathbf{b}_{3}^{\prime}$ & $\mathbf{a}$ & $\mathbf{a}^{\prime}$ \\
\hline $\mathbf{1}$ & 0 & 0 & 1 & 1 & 1 & 1 & 1 & 1 & 0 & 0 \\
$\mathbf{S}$ & 0 & 0 & 0 & 0 & 0 & 0 & 0 & 0 & 0 & 0 \\
\hline $\mathbf{b}_{1}$ & 1 & 1 & 1 & 1 & 1 & 0 & 0 & 0 & 0 & 0 \\
$\mathbf{b}_{2}$ & 1 & 1 & 1 & 1 & 1 & 0 & 0 & 0 & 0 & 0 \\
$\mathbf{b}_{3}$ & 1 & 1 & 1 & 1 & 1 & 0 & 0 & 0 & 0 & 0 \\
\hline $\mathbf{b}_{1}^{\prime}$ & 1 & 1 & 0 & 0 & 0 & 1 & 1 & 1 & 0 & 0 \\
$\mathbf{b}_{2}^{\prime}$ & 1 & 1 & 0 & 0 & 0 & 1 & 1 & 1 & 0 & 0 \\
$\mathbf{b}_{3}^{\prime}$ & 1 & 1 & 0 & 0 & 0 & 1 & 1 & 1 & 0 & 0 \\
\hline $\mathbf{a}$ & 0 & 0 & 1 & 1 & 1 & 0 & 0 & 0 & 1 & $* 1$ \\
$\mathbf{a}^{\prime}$ & 0 & 0 & 0 & 0 & 0 & 1 & 1 & 1 & $* 0$ & 1 \\
\hline
\end{tabular}

Table C.2 Model 2 Gauge Group

\begin{tabular}{|l|rrrrr|}
\hline Observable & $\psi^{1}$ & $\psi^{2}$ & $\psi^{3}$ & $\psi^{3}$ & $\psi^{5}$ \\
\hline \hline$S U(4)_{C}$ & 0 & 1 & -1 & 0 & 0 \\
& 1 & 0 & -1 & 0 & 0 \\
& 0 & 1 & 1 & 0 & 0 \\
\hline$S U(2)_{R}$ & 0 & 0 & 0 & 1 & 1 \\
\hline
\end{tabular}

\begin{tabular}{|c|rrr|rrrrrr|rrr|}
\hline Shadow & $\bar{\eta}^{1}$ & $\bar{\eta}^{2}$ & $\bar{\eta}^{3}$ & $\bar{y}_{1,3}$ & $\bar{y}_{2,4}$ & $\bar{y}_{5} \bar{w}_{1}$ & $\bar{y}_{6} \bar{\omega}_{2}$ & $\bar{\omega}_{3,5}$ & $\bar{\omega}_{4,6}$ & $\bar{\eta}^{\prime}$ & $\bar{\eta}^{\prime 2}$ & $\bar{\eta}^{\prime}$ \\
\hline \hline$S U(2)^{3}$ & $\frac{1}{2}$ & $-\frac{1}{2}$ & 0 & 0 & $\frac{1}{2}$ & 0 & $-\frac{1}{2}$ & $-\frac{1}{2}$ & $\frac{1}{2}$ & $-\frac{1}{2}$ & $\frac{1}{2}$ & 0 \\
& $\frac{1}{2}$ & 0 & $-\frac{1}{2}$ & $\frac{1}{2}$ & 0 & $-\frac{1}{2}$ & $\frac{1}{2}$ & 0 & $-\frac{1}{2}$ & $-\frac{1}{2}$ & 0 & $\frac{1}{2}$ \\
& 0 & $\frac{1}{2}$ & $-\frac{1}{2}$ & $-\frac{1}{2}$ & $\frac{1}{2}$ & $\frac{1}{2}$ & 0 & $-\frac{1}{2}$ & 0 & 0 & $-\frac{1}{2}$ & $\frac{1}{2}$ \\
\hline$S U(3)$ & $\frac{1}{2}$ & $-\frac{1}{2}$ & 0 & 0 & $-\frac{1}{2}$ & 0 & $\frac{1}{2}$ & $-\frac{1}{2}$ & $\frac{1}{2}$ & $\frac{1}{2}$ & $-\frac{1}{2}$ & 0 \\
& $\frac{1}{2}$ & 0 & $-\frac{1}{2}$ & $-\frac{1}{2}$ & 0 & $-\frac{1}{2}$ & $\frac{1}{2}$ & 0 & $\frac{1}{2}$ & $\frac{1}{2}$ & 0 & $-\frac{1}{2}$ \\
\hline
\end{tabular}

\begin{tabular}{|c|rrrrr|rrrrr|}
\hline Hidden & $\bar{\psi}^{1}$ & $\bar{\psi}^{2}$ & $\bar{\psi}^{3}$ & $\bar{\psi}^{3}$ & $\bar{\psi}^{5}$ & $\bar{\psi}^{1}$ & $\bar{\psi}^{\prime 2}$ & $\bar{\psi}^{3}$ & $\bar{\psi}^{3}$ & $\bar{\psi}^{5}$ \\
\hline \hline$S O(10)$ & 0 & 0 & 0 & 0 & 0 & 0 & 0 & 0 & 1 & 1 \\
& 0 & 0 & 0 & $\frac{1}{2}$ & $-\frac{1}{2}$ & 0 & 0 & -1 & $\frac{1}{2}$ & $\frac{1}{2}$ \\
& 0 & 0 & 0 & 0 & 0 & 0 & 1 & -1 & 0 & 0 \\
& 0 & 0 & 0 & 0 & 0 & 1 & -1 & 0 & 0 & 0 \\
& 0 & 0 & 0 & 0 & 0 & 1 & 1 & 0 & 0 & 0 \\
\hline$S U(2)_{L}$ & 0 & 0 & 0 & 0 & 0 & 0 & 0 & 0 & 1 & -1 \\
\hline
\end{tabular}


Table C.3 Model 2 Left-Handed States

\begin{tabular}{|c|c|c|c|c|c|c|c|c|c|c|}
\hline \multirow{2}{*}{\begin{tabular}{|l}
$n=1,2$ \\
Singlets
\end{tabular}} & \multicolumn{3}{|c|}{$\begin{array}{lll}\left(4_{C}, 2_{R}\right)_{O} & \left(2^{3}, 3\right)^{S} & \left(10,2_{R}\right)^{H}\end{array}$} & $4 Q_{1}$ & $4 Q_{2}$ & $4 Q_{3}$ & $4 Q_{4}$ & \multicolumn{3}{|c|}{$\begin{array}{lll}4 Q_{5} & 4 Q_{6} & 4 Q_{7} \\
\end{array}$} \\
\hline & & & & & & & & & & \\
\hline$S_{1}^{n}$ & $(1,1)$ & $(1,1,1,1)$ & $(1,1)$ & 12 & 0 & -4 & -8 & 16 & 0 & 0 \\
\hline$S_{2}^{n}$ & $(1,1)$ & $(1,1,1,1)$ & $(1,1)$ & 12 & 0 & -4 & 8 & -16 & 0 & 0 \\
\hline$S_{3}^{n}$ & $(1,1)$ & $(1,1,1,1)$ & $(1,1)$ & 12 & 0 & 0 & -6 & 0 & 4 & 24 \\
\hline$S_{4}^{n}$ & $(1,1)$ & $(1,1,1,1)$ & $(1,1)$ & 12 & 0 & 0 & 6 & 0 & 4 & 24 \\
\hline$S_{5}^{n}$ & $(1,1)$ & $(1,1,1,1)$ & $(1,1)$ & 12 & 0 & 0 & 2 & -16 & 4 & -24 \\
\hline$S_{6}^{n}$ & $(1,1)$ & $(1,1,1,1)$ & $(1,1)$ & 12 & 0 & 0 & -2 & 16 & 4 & -24 \\
\hline$S_{7}$ & $(1,1)$ & $(1,1,1,1)$ & $(1,1)$ & 0 & 0 & 0 & 8 & 32 & 0 & 0 \\
\hline $\bar{S}_{7}$ & $(1,1)$ & $(1,1,1,1)$ & $(1,1)$ & 0 & 0 & 0 & -8 & -32 & 0 & 0 \\
\hline$S_{8}$ & $(1,1)$ & $(1,1,1,1)$ & $(1,1)$ & 0 & 0 & 8 & 4 & 16 & 8 & 0 \\
\hline $\bar{S}_{8}$ & $(1,1)$ & $(1,1,1,1)$ & $(1,1)$ & 0 & 0 & -8 & -4 & -16 & -8 & 0 \\
\hline$S_{9}$ & $(1,1)$ & $(1,1,1,1)$ & $(1,1)$ & 0 & 0 & 8 & -4 & -16 & 8 & 0 \\
\hline $\bar{S}_{9}$ & $(1,1)$ & $(1,1,1,1)$ & $(1,1)$ & 0 & 0 & -8 & 4 & 16 & -8 & 0 \\
\hline Observab & & & & & & & & & & \\
\hline$Q L_{1}^{n}$ & $\overline{4,2)}$ & $(1,1,1,1)$ & $(1,1)$ & -6 & -2 & -2 & 2 & -16 & $\overline{0}$ & -8 \\
\hline$Q L_{2}^{n}$ & $4,2)$ & $(1,1$, & $(1,1)$ & -6 & -2 & -2 & -2 & 16 & 0 & -8 \\
\hline$Q L_{3}^{n}$ & $(4,2)$ & $(1,1$, & $(1,1)$ & -6 & -2 & 2 & 0 & 0 & 4 & 16 \\
\hline$\left(q^{c} l^{c}\right)_{1}^{n}$ & $(-4,1)$ & $(2,1,1)$ & $(1,1)$ & 6 & -6 & -2 & 0 & 0 & -4 & 0 \\
\hline$\left(q^{c} l^{c}\right)_{2}^{n}$ & $(-4,1)$ & $(1,2,1$, & $(1,1)$ & 6 & -6 & 2 & -2 & -8 & 0 & 0 \\
\hline$\left(q^{c} l^{c}\right)_{3}^{n}$ & $(-4,1)$ & $(1,1,2,1)$ & $(1,1)$ & 6 & -6 & 2 & 2 & 8 & 0 & 0 \\
\hline$h_{1}$ & $(1,2)$ & $(2,1,1,1)$ & $(1,1)$ & $\overline{0}$ & -8 & $\overline{0}$ & 0 & 0 & $\overline{0}$ & 16 \\
\hline $\bar{h}_{1}$ & $(1,2)$ & $(2,1,1,1)$ & $(1,1)$ & 0 & 8 & 0 & 0 & 0 & 0 & -16 \\
\hline$h_{2}$ & $(1,2)$ & $(1,2,1,1)$ & $(1,1)$ & 0 & -8 & 0 & -4 & 8 & 0 & -8 \\
\hline $\bar{h}_{2}$ & $(1,2)$ & $(1,2,1,1$ & $(1,1)$ & 0 & 8 & 0 & 4 & -8 & 0 & 8 \\
\hline$h_{3}$ & $(1,2)$ & $(1,1,2,1)$ & $(1,1)$ & 0 & -8 & 0 & 4 & -8 & 0 & -8 \\
\hline $\bar{h}_{3}$ & $(1,2)$ & $(1,1,2,1)$ & $(1,1)$ & 0 & 8 & 0 & -4 & 8 & 0 & 8 \\
\hline$H_{1}^{n}$ & $(1,2)$ & $(2,1,1,3)$ & $(1,1)$ & 4 & 0 & 4 & 0 & 0 & 0 & 0 \\
\hline$H_{2}^{n}$ & $(1,2)$ & $(1,2,1,3)$ & $(1,1)$ & 4 & 0 & 0 & 2 & 8 & -4 & 0 \\
\hline$H_{3}^{n}$ & $(1,2)$ & $(1,1,2,3)$ & $(1,1)$ & 4 & 0 & 0 & -2 & -8 & -4 & 0 \\
\hline$B_{1}^{n}$ & $(6,1)$ & $(1,1,1,1)$ & $(1,1)$ & 0 & 4 & 4 & 6 & 0 & -4 & -8 \\
\hline$B_{2}^{n}$ & $(6,1)$ & $(1,1,1,1)$ & $(1,1)$ & 0 & 4 & 0 & 0 & 0 & -8 & 16 \\
\hline$B_{3}^{n}$ & $(6,1)$ & $(1,1,1,1)$ & $(1,1)$ & 0 & 4 & 4 & -6 & 0 & -4 & -8 \\
\hline
\end{tabular}


Table C.3 Model 2 Left-Handed States cont.

\begin{tabular}{|c|c|c|c|c|c|c|c|c|c|c|}
\hline$n=1,2$ & $\left(4_{C}, 2_{R}\right)_{O}$ & $\left(2^{3}, 3\right)^{S}$ & $\left(10,2_{R}\right)^{H}$ & $4 Q_{1}$ & $4 Q_{2}$ & $4 Q_{3}$ & $4 Q_{4}$ & $4 Q_{5}$ & $4 Q_{6}$ & $4 Q_{7}$ \\
\hline \multicolumn{11}{|l|}{ Shadow } \\
\hline$F_{1}^{n}$ & $(1,1)$ & $(1,1,1, \overline{3})$ & $(1,1)$ & -4 & -8 & -4 & 0 & 0 & 0 & 16 \\
\hline$F_{2}^{n}$ & $(1,1)$ & $(1,1,1, \overline{3})$ & $(1,1)$ & -4 & -8 & 0 & -6 & 0 & 4 & -8 \\
\hline$F_{3}^{n}$ & $(1,1)$ & $(1,1,1, \overline{3})$ & $(1,1)$ & -4 & -8 & 0 & 6 & 0 & 4 & -8 \\
\hline$F_{4}^{n}$ & $(1,1)$ & $(1,1,1, \overline{3})$ & $(1,1)$ & -4 & 8 & -4 & 0 & 0 & 0 & -16 \\
\hline$F_{5}^{n}$ & $(1,1)$ & $(1,1,1, \overline{3})$ & $(1,1)$ & -4 & 8 & 0 & -2 & 16 & 4 & 8 \\
\hline$F_{6}^{n}$ & $(1,1)$ & $(1,1,1, \overline{3})$ & $(1,1)$ & -4 & 8 & 0 & 2 & -16 & 4 & 8 \\
\hline$F_{7}^{n}$ & $(1,1)$ & $(1,1,1,3)$ & $(1,1)$ & -8 & 0 & -8 & 0 & 0 & 0 & 0 \\
\hline $\bar{F}_{7}^{n}$ & $(1,1)$ & $(1,1,1, \overline{3})$ & $(1,1)$ & 8 & 0 & 8 & 0 & 0 & 0 & 0 \\
\hline$F_{8}^{n}$ & $(1,1)$ & $(1,1,1,3)$ & $(1,1)$ & -8 & 0 & 0 & -4 & -16 & 8 & 0 \\
\hline $\bar{F}_{8}^{n}$ & $(1,1)$ & $(1,1,1, \overline{3})$ & $(1,1)$ & 8 & 0 & 0 & 4 & 16 & -8 & 0 \\
\hline$F_{9}^{n}$ & $(1,1)$ & $(1,1,1,3)$ & $(1,1)$ & -8 & 0 & 0 & 4 & 16 & 8 & 0 \\
\hline $\bar{F}_{9}^{n}$ & $(1,1)$ & $(1,1,1, \overline{3})$ & $(1,1)$ & 8 & 0 & 0 & -4 & -16 & -8 & 0 \\
\hline$X_{1}^{n}$ & $(1,1)$ & $(2,2,1,1)$ & $(1,1)$ & -12 & 0 & 0 & -2 & -8 & -4 & 0 \\
\hline$X_{2}$ & $(1,1)$ & $(2,2,1,1)$ & $(1,1)$ & 0 & 0 & 0 & -4 & 8 & 0 & -24 \\
\hline$X_{3}$ & $(1,1)$ & $(2,2,1,1)$ & $(1,1)$ & 0 & 0 & 0 & 4 & -8 & 0 & 24 \\
\hline$Y_{1}^{n}$ & $(1,1)$ & $(2,1,2,1)$ & $(1,1)$ & -12 & 0 & 0 & 2 & 8 & -4 & 0 \\
\hline$Y_{2}$ & $(1,1)$ & $(2,1,2,1)$ & $(1,1)$ & 0 & 0 & 0 & 4 & -8 & 0 & -24 \\
\hline$Y_{3}$ & $(1,1)$ & $(2,1,2,1)$ & $(1,1)$ & 0 & 0 & 0 & -4 & 8 & 0 & 24 \\
\hline$Z_{1}^{n}$ & $(1,1)$ & $(1,2,2,1)$ & $(1,1)$ & -12 & 0 & 4 & 0 & 0 & 0 & 0 \\
\hline$Z_{2}$ & $(1,1)$ & $(1,2,2,1)$ & $(1,1)$ & 0 & 0 & 0 & -8 & 16 & 0 & 0 \\
\hline$Z_{3}$ & $(1,1)$ & $(1,2,2,1)$ & $(1,1)$ & 0 & 0 & 0 & 8 & -16 & 0 & 0 \\
\hline \multicolumn{11}{|l|}{ Hidden } \\
\hline$T_{1}^{n}$ & $(1,1)$ & $(1,1,1,1)$ & $(10,1)$ & 0 & -4 & 4 & -2 & 16 & -4 & 8 \\
\hline$T_{2}^{n}$ & $(1,1)$ & $(1,1,1,1)$ & $(10,1)$ & 0 & -4 & 4 & 2 & -16 & -4 & 8 \\
\hline$T_{3}^{n}$ & $(1,1)$ & $(1,1,1,1)$ & $(10,1)$ & 0 & -4 & 0 & 0 & 0 & -8 & -16 \\
\hline$F_{1}^{n}$ & $(1,1)$ & $(1,1,1, \overline{3})$ & $(1,2)$ & -4 & -4 & 4 & 0 & 0 & 0 & -16 \\
\hline $\bar{F}_{2}^{n}$ & $(1,1)$ & $(1,1,1, \overline{3})$ & $(1,2)$ & -4 & -4 & 0 & -6 & 0 & -4 & 8 \\
\hline $\bar{F}_{3}^{n}$ & $(1,1)$ & $(1,1,1, \overline{3})$ & $(1,2)$ & -4 & -4 & 0 & 6 & 0 & -4 & 8 \\
\hline$D_{1}^{n}$ & $(1,1)$ & $(1,1,1,1)$ & $(1,2)$ & 12 & 4 & 4 & 0 & 0 & 0 & 16 \\
\hline$D_{2}^{n}$ & $(1,1)$ & $(1,1,1,1)$ & $(1,2)$ & 12 & 4 & 0 & -2 & 16 & -4 & -8 \\
\hline$D_{3}^{n}$ & $(1,1)$ & $(1,1,1,1)$ & $(1,2)$ & 12 & 4 & 0 & 2 & -16 & -4 & -8 \\
\hline
\end{tabular}


Table D.1 Hypercharge Components.

\begin{tabular}{|l|rrr|ll|}
\hline & $\begin{array}{r}\tilde{Q}_{B-L} \\
\frac{1}{3} \tilde{Q}_{C}\end{array}$ & $\frac{1}{2} \tilde{Q}_{L}^{R}$ & $2 T_{3}^{L}$ & $\tilde{Q}_{E M}$ & $\begin{array}{l}=T_{3}^{L}+T_{3}^{R}+\frac{1}{2} \tilde{Q}_{B-L} \\
=T_{3}^{L}+\frac{1}{2} Y\end{array}$ \\
\hline \hline$Q_{L}$ & $\frac{1}{3}$ & 0 & \pm 1 & $\frac{2}{3},-\frac{1}{3}$ \\
$d_{L}^{c}$ & $-\frac{1}{3}$ & 1 & 0 & $\frac{1}{3}$ \\
$u_{L}^{c}$ & $-\frac{1}{3}$ & -1 & 0 & $-\frac{2}{3}$ \\
$L_{L}$ & -1 & 0 & \pm 1 & $0,-1$ \\
$e_{L}^{c}$ & 1 & 1 & 0 & 1 \\
$\nu_{L}^{c}$ & 1 & -1 & 0 & 0 \\
\hline
\end{tabular}

